\title{
Soluble urokinase plasminogen activator receptor for the prediction of ventilator-associated pneumonia
}

\author{
Pouline M. van Oort (101, Lieuwe D. Bos (10 ${ }^{1}$, Pedro Póvoa ${ }^{2,3}$, Paula Ramirez ${ }^{4}$, \\ Antoni Torres ${ }^{5,6}$, Antonio Artigas ${ }^{6,7,8}$, Marcus J. Schultz ${ }^{1,9}$ and \\ Ignacio Martin-Loeches ${ }^{6,10}$ on behalf of the BioVAP study
}

Affiliations: ${ }^{1}$ Dept of Intensive Care, Amsterdam UMC, Academic Medical Centre, Amsterdam, The Netherlands. ${ }^{2}$ Polyvalent Intensive Care Unit, São Francisco Xavier Hospital, Centro Hospitalar de Lisboa Ocidental, Lisbon, Portugal. ${ }^{3}$ NOVA Medical School, New University of Lisbon, Lisbon, Portugal. ${ }^{4}$ Respiratory Disease Dept, Hospital Clínici Provincial de Barcelona, IDIBAPS, Barcelona, Spain. ${ }^{5}$ Intensive Care Unit, University Hospital La Fe, Valencia, Spain. ${ }^{6}$ CIBER de Enfermedades Respiratorias, Centro de Investigación Biomédica en Red, Madrid, Spain. ${ }^{7}$ Critical Care Dept, Corporacion Sanitaria Universitaria Parc Tauli, Autonomous University of Barcelona, Sabadell, Spain. ${ }^{8}$ Critical Care Dept, Sagrado Corazon-General de Catalunya University Hospitals, Quiron Salud-IDC, Barcelona, Spain. ${ }^{9}$ Mahidol-Oxford Tropical Medicine Research Unit (MORU), Mahidol University, Bangkok, Thailand. ${ }^{10}$ Dept of Clinical Medicine, St James's University Hospital, Dublin, Ireland.

Correspondence: Pouline M. van Oort, Dept of Intensive Care, Amsterdam UMC, Academic Medical Centre, Meibergdreef 9, 1105 AZ Amsterdam, The Netherlands. E-mail: p.m.vanoortdamc.uva.nl

\section{ABSTRACT}

Introduction: Diagnosing ventilator-associated pneumonia (VAP) remains challenging. Soluble urokinase plasminogen activator receptor (suPAR) has prognostic value in critically ill patients with systemic infection. We hypothesised that plasma suPAR levels accurately predict development of VAP.

Methods: This observational, multicentre, prospective cohort study compared patients at risk for VAP with a control group. Plasma and tracheal aspirate samples were collected. Plasma suPAR levels were measured on the day of diagnosis and 3 days before diagnosis.

Results: The study included 24 VAP patients and 19 control patients. The suPAR concentration measured 3 days before diagnosis was significantly increased in VAP patients versus matched samples of control patients (area under the receiver operating characteristic curve (AUC) 0.68, 95\% CI 0.52-1.00; $\mathrm{p}=0.04$ ). Similar results were found on the day of diagnosis (AUC 0.77, 95\% CI 0.6-0.93; $\mathrm{p}=0.01$ ). Plasma suPAR was significantly higher in deceased patients (AUC 0.79, 95\% CI 0.57-1.00; $\mathrm{p}<0.001$ ). Combining suPAR with the Clinical Pulmonary Infection Score, C-reactive protein and/or procalcitonin led to a significantly increased discriminative accuracy for predicting VAP and an increased specificity.

Conclusions: suPAR can be used to diagnose VAP with a fair diagnostic accuracy and has a moderate prognostic accuracy to be used in critically ill intensive care unit patients. Its performance improves when added to other clinically available biomarkers (C-reactive protein and procalcitonin) or scoring systems (Clinical Pulmonary Infection Score and Sepsis-related Organ Failure Assessment).

$@ E R S p u b l i c a t i o n s$

suPAR can be used to diagnose VAP with a fair diagnostic accuracy and has a moderate prognostic accuracy to be used in critically ill ICU patients http://ow.ly/ubmf30nIgZO

Cite this article as: van Oort PM, Bos LD, Póvoa $\mathrm{P}$, et al. Soluble urokinase plasminogen activator receptor for the prediction of ventilator-associated pneumonia. ERJ Open Res 2019; 5: 00212-2018 [https://doi.org/10.1183/23120541.00212-2018]. 


\section{Introduction}

Ventilator-associated pneumonia (VAP) is accountable for approximately one-third of nosocomial pneumonia cases [1], associated with a longer intensive care unit (ICU) and hospital length of stay (LOS), and increased healthcare costs [2]. VAP is associated with significant mortality [3, 4], excess morbidity [5] and an increased duration of mechanical ventilation $[1,6]$.

Due to the absence of a clinically available gold standard, the diagnosis of VAP remains challenging. The clinician has to rely on clinical parameters and radiological, laboratory and microbiological results [7], which may take hours to days to become available. A quicker predictive test is urgently needed, enabling a more accurate prediction of pneumonia and thus limiting antimicrobial misuse.

Multiple biomarkers, measured in serum and in bronchoalveolar lavage fluid (BALF), have been investigated for their diagnostic value in infections, e.g. the soluble triggering receptor expressed on myeloid cells sTREM-1 [8], interleukin (IL)-1 $\beta$ and IL-8 [9-11], C-reactive protein (CRP), and procalcitonin (PCT) $[12,13]$. As yet, none of these biomarkers have been proven to have sufficient diagnostic or prognostic quality to be used in patients receiving antibiotic treatment for VAP [1]. Soluble urokinase plasminogen activator receptor (suPAR) has become of interest as an inflammatory biomarker that seems to positively correlate with the activity of the immune system and has been shown to have prognostic value in critically ill patients with systemic infection in terms of mortality prediction $[14,15]$. In patients with acute respiratory distress syndrome, significantly higher suPAR plasma levels were found with increasing disease severity [16]. In critically ill patients, higher plasma suPAR levels were associated with longer duration of mechanical ventilation and increased ICU LOS [17], a higher incidence rate of readmission, and an increased mortality rate [18, 19]. In VAP and sepsis patients, suPAR has been shown to be an independent predictor of an unfavourable outcome [20].

We hypothesised that suPAR measured in plasma of critically ill patients can predict VAP 3 days before clinical symptoms occur, and we postulate that we will confirm the prognostic value of suPAR for mortality and ICU LOS. For this purpose, we performed a post hoc analysis of carefully defined VAP cases and matched controls recruited in the BioVAP study.

\section{Methods}

Study design and ethical approval

This was a post hoc study of the investigator-initiated observational, multicentre, prospective BioVAP (Biomarkers in the diagnosis and management of Ventilator-Associated Pneumonia) study, a study that ran in the ICUs of four university teaching hospitals [12]. BioVAP was registered at ClinicalTrials.gov (identifier NCT02078999), and the study protocol was approved by the institutional review boards and the local ethics committees of the four participating hospitals. Written informed consent was obtained from all patients or their legally authorised proxies.

\section{Study population}

Consecutive patients admitted to a participating ICU were screened for eligibility for recruitment over a 3 -year period. Only the first ICU admission and the first VAP episode were included in the study. Patients were included when 1) they were on mechanical ventilation for $>72 \mathrm{~h}, 2$ ) there were no signs of pneumonia on the chest radiograph at admission, 3) they had not been receiving antibiotic treatment within 5 days prior to admission to the ICU, 4) expected duration of mechanical ventilation was $>72 \mathrm{~h}$ and 5) no antibiotics were prescribed on admission by the responsible clinician (except for prophylactic purposes).

Exclusion criteria involved current or past participation in an intervention trial conflicting with the BioVAP study (e.g. studies into mechanical ventilation, antibiotic therapy or VAP prophylaxis), previous endotracheal intubation of $>12 \mathrm{~h}$ within 30 days prior to recruitment, body mass index $>40 \mathrm{~kg} \cdot \mathrm{m}^{-2}$, age $<18$ years, pregnancy, patients with known bronchiectasis, cystic fibrosis and/or witnessed pulmonary aspiration prior to or at intubation, and patients from whom informed consent was not obtained.

The control group consisted of ICU patients that were mechanically ventilated for $>7$ days, but did not develop VAP. This cut-off was selected because it corresponds to the median day of VAP development for the cases.

\section{Primary and secondary end-points}

The primary end-point was the diagnostic accuracy of suPAR for the diagnosis of VAP. The secondary end-points were the predictive accuracy of suPAR regarding mortality and ICU LOS. 
Definition of VAP

VAP was defined as: a respiratory infection developing at least $48 \mathrm{~h}$ after the start of invasive mechanical ventilation in patients that had no signs of pneumonia at the time of tracheal intubation and without signs of pneumonia on their chest radiograph at admission to the ICU. The definition of VAP was based either on 1) clinical criteria (new or progressive pulmonary infiltrates on chest radiographs, together with at least two of the following: temperature $>38^{\circ} \mathrm{C}$ or $<36^{\circ} \mathrm{C}$, white blood cell count $>10000$ or $<4000 \mathrm{~mm}^{-3}$, or purulent sputum) or 2) a simplified Clinical Pulmonary Infection Score (CPIS) $\geqslant 6$ (after $48 \mathrm{~h}$ of mechanical ventilation) [21]. Pneumonia was microbiologically confirmed by the presence of at least one potentially pathogenic microorganism in respiratory samples above certain predefined thresholds: for BALF samples $>10^{4} \mathrm{CFU} \cdot \mathrm{mL}^{-1}$ and for sputum or tracheobronchial aspirate samples $>10^{5} \mathrm{CFU} \cdot \mathrm{mL}^{-1}$.

\section{Follow-up}

Follow-up of patients was established up until day 21 after ICU admission, the day of successful weaning and extubation, the day of an ICU-acquired infection other than VAP or the day of clinical diagnosis of VAP. Death, ICU discharge and hospital discharge were also assessed. A telephone interview was performed on day 90 after ICU admission in order to assess outcome.

\section{Sample collection and measurements}

Blood samples were obtained from an arterial line once daily from the time of ICU admission onwards and throughout the period of mechanical ventilation. suPAR was measured in the samples taken on the day of diagnosis, as well as in the samples taken 3 days before diagnosis. For the non-VAP patients (i.e. the control group) the samples were obtained in parallel: samples matched when they were collected after the same amount of days after exposure to the risk factor (i.e. start of mechanical ventilation).

The blood samples were collected using sterile Vacutainer tubes (BD, Franklin Lakes, NJ, USA) containing citrate and were centrifuged for $10 \mathrm{~min}$ at $4^{\circ} \mathrm{C}$ at $1800 \times g$. suPAR detection was performed by ELISA using the suPARnostic kit provided by ViroGates (Birkerød, Denmark), which can determine suPAR concentrations as $\mathrm{ng} \cdot \mathrm{mL}^{-1}$ plasma.

A quantitative tracheal aspirate was obtained for all patients: the first sample was collected on ICU admission and subsequently twice a week (Mondays and Thursdays or Tuesdays and Fridays). These samples were used for microbiological confirmation of VAP.

\section{Statistical analysis}

Data are shown as median (interquartile range (IQR)). Plasma suPAR levels were compared between groups (patients who developed VAP versus those that did not develop VAP) using the nonparametric Mann-Whitney U-test. This was done for plasma suPAR measured at the time of diagnosis, as well as 3 days before diagnosis. For the control patients, matched samples were used that were collected after the same amount of days after the start of mechanical ventilation. The area under the receiver operating characteristic (ROC) curve (AUC) was used to assess the discriminative ability, sensitivity and specificity of suPAR levels: an AUC of 0.6-0.7 was considered as poor, 0.7-0.8 as fair, 0.8-0.9 as good and 0.9-1.0 as excellent. The default "DeLong method" in the roc.test function in R statistics was used to compare AUCs [22]. A p-value $<0.05$ was considered statistically significant. Linear regression was performed to assess the relation between hospital LOS and ICU LOS. Statistical analyses were performed in R statistics via the R-studio interface [23].

\section{Results}

Data and samples were collected from 43 patients (table 1); a flowchart detailing patient inclusion is shown in figure 1. VAP was microbiologically confirmed in 24 patients, which were considered cases; 19 noninfected patients served as controls. Simplified Acute Physiology Score II (SAPS II) [24] and Sepsis-related Organ Failure Assessment (SOFA) [25] scores were significantly higher between cases and controls. In addition, ICU LOS differed significantly in cases versus controls $(\mathrm{p}<0.001)$, as did the mortality rate $(\mathrm{p}=0.001)$.

\section{Microbiology of VAP}

For the 24 VAP patients, a total of 27 isolates were distinguished in the respiratory samples: methicillin-susceptible Staphylococcus aureus (six patients), Haemophilus influenzae (four patients), Escherichia coli (three patients), Klebsiella pneumoniae (three patients), methicillin-resistant S. aureus (two patients), Pseudomonas aeruginosa (two patients), Citrobacter spp. (two patients), Streptococcus spp. (one patient), Acinetobacter baumannii (one patient), Moraxella catarrhalis (one patient), Proteus spp. (one patient) and Enterobacteriaceae spp. (one patient). The respiratory sample contained more than one pathogen in three cases. 


\section{TABLE 1 Patient characteristics}

\begin{tabular}{|c|c|c|c|}
\hline & Control patients & VAP patients & p-value \\
\hline Subjects & 19 & 24 & \\
\hline Age years & $49.89 \pm 23.51$ & $53.96 \pm 16.79$ & 0.53 \\
\hline Male & $10(52.6)$ & 18 (75) & 0.21 \\
\hline COPD & $0(0)$ & $4(16.7)$ & 0.12 \\
\hline Diabetes & $2(10.5)$ & $2(8.3)$ & 1.0 \\
\hline Immunosuppression & $0(0)$ & $1(4.2)$ & 1.0 \\
\hline Heart failure & $2(10.5)$ & $1(4.2)$ & 0.58 \\
\hline Liver failure & $0(0)$ & $1(4.2)$ & 1.0 \\
\hline Renal failure & $1(5.3)$ & $2(8.3)$ & 1.0 \\
\hline APACHE II score & $22.0(17.50-24.50)$ & $25.5(20.75-33.00)$ & 0.11 \\
\hline SAPS II score & $44(23.50-50.50)$ & $57(46.75-71.00)$ & 0.004 \\
\hline SOFA score & $6(5.00-8.00)$ & $8(6.00-11.00)$ & 0.04 \\
\hline WBC count $\mathrm{mm}^{-3}$ & 10.4 (8.30-14.27) & $14.5(9.59-16.00)$ & 0.15 \\
\hline CPIS score & $3(1.00-4.00)$ & $3(1.00-3.50)$ & 0.59 \\
\hline ICU LOS days & $8.0(6.00-10.00)$ & $19.5(14.00-23.75)$ & $<0.001$ \\
\hline Hospital LOS days & $21(15.50-24.00)$ & $30(20.00-43.25)$ & 0.08 \\
\hline ICU mortality & $0(0)$ & $10(41.7)$ & 0.001 \\
\hline \multicolumn{4}{|c|}{$\begin{array}{l}\text { Data are presented as } \mathrm{n} \text {, mean } \pm \mathrm{SD}, \mathrm{n}(\%) \text { or median (interquartile range), unless otherwise stated. VAP: } \\
\text { ventilator-associated pneumonia; COPD: chronic obstructive pulmonary disease; APACHE II: Acute } \\
\text { Physiology and Chronic Health Evaluation II; SAPS II: Simplified Acute Physiology Score II; SOFA: } \\
\text { Sepsis-related Organ Failure Assessment; WBC: white blood cell; CPIS: Clinical Pulmonary Infection } \\
\text { Score; ICU: intensive care unit; LOS: length of stay. }\end{array}$} \\
\hline
\end{tabular}

Accuracy of sUPAR levels for prediction of VAP 3 days before diagnosis

suPAR levels were significantly higher in the plasma of confirmed VAP patients (median (IQR) 6.2 (4.2-12.2) ng. $\mathrm{mL}^{-1}$ ) compared with controls $\left(4.5(3.5-5.9) \mathrm{ng} \cdot \mathrm{mL}^{-1}\right)$ when measured 3 days before diagnosis (AUC 0.68, 95\% CI 0.52-1.00; $\mathrm{p}=0.04$ ) (figure 2).

When measured 3 days before diagnosis, the AUC for CPIS alone was 0.63 (95\% CI 0.46-0.80; $\mathrm{p}=0.16$ ). The combination of CPIS and suPAR led to an AUC of 0.68 (95\% CI 0.52-0.84). The difference between the two ROC curves was not significant $(p=0.54)$. The net reclassification improvement was 0.06 (or $6 \%$; $\mathrm{p}=0.005)$, resulting in a higher specificity. CRP measured 3 days before diagnosis showed an AUC of 0.68 (95\% CI $0.50-0.86 ; \mathrm{p}=0.04)$. The combination of CRP and suPAR had an AUC of 0.74 (95\% CI $0.56-0.92)$. The difference between these two AUCs was not significant $(p=0.36)$. The net reclassification index (NRI) was 0.07 (7\%; $\mathrm{p}=0.17)$, leading to an increased specificity. A weak positive correlation was found between suPAR and CRP, with a correlation coefficient of 0.21 .

PCT had an AUC of 0.73 (95\% CI 0.55-0.91; $\mathrm{p}=0.06$ ) when measured 3 days before diagnosis. PCT combined with suPAR resulted in an AUC of 0.81 (95\% CI 0.64-0.97). Again, the difference between the

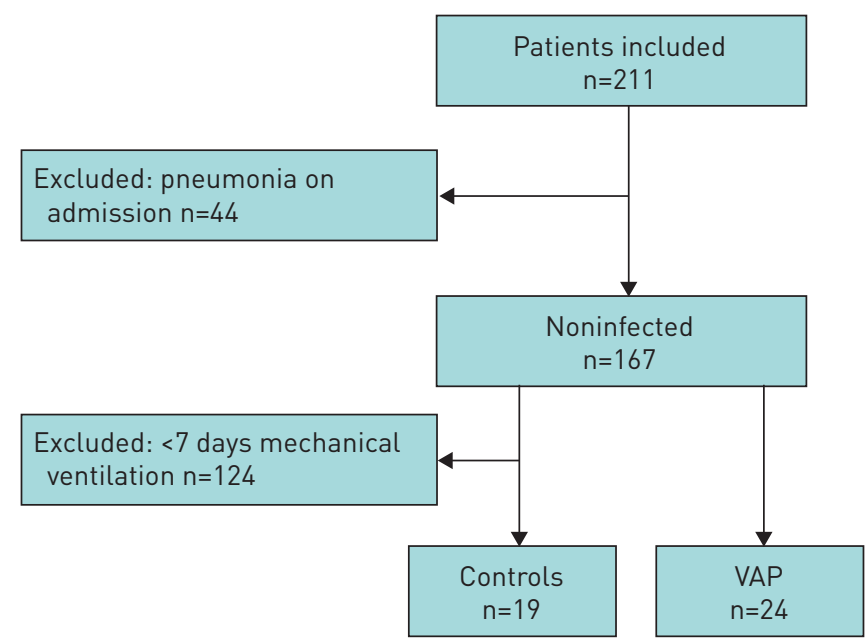

FIGURE 1 Flowchart of patient inclusion. 


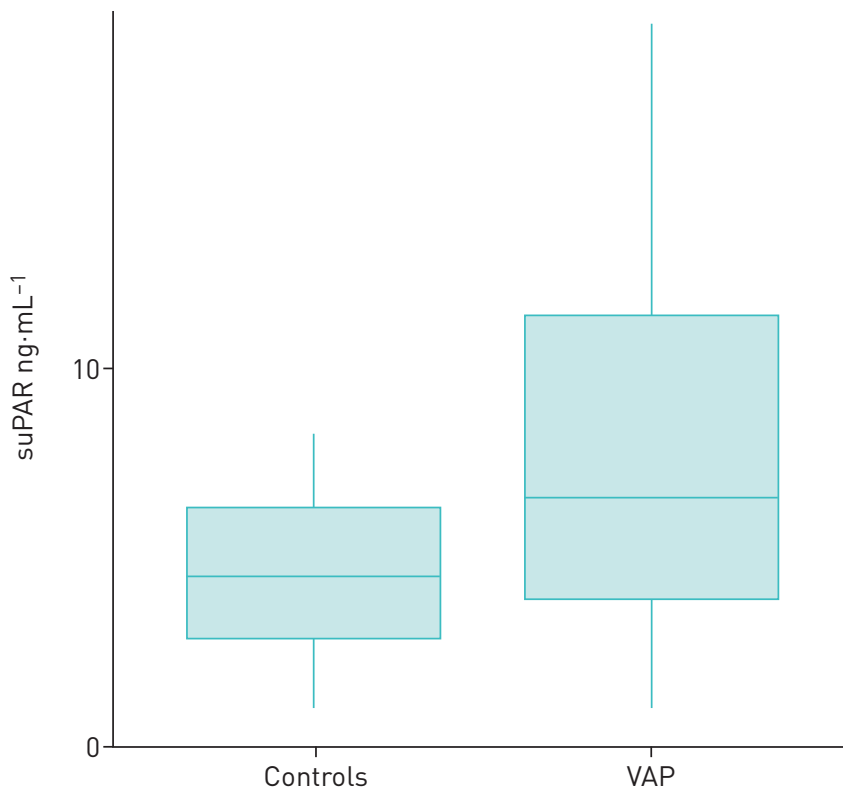

FIGURE 2 Difference in soluble urokinase plasminogen activator receptor (suPAR) levels between diagnosed ventilator-associated pneumonia (VAP) patients and controls, measured 3 days before VAP diagnosis. Data are presented as box-and-whisker plots, with boxes showing the median (interquartile range (IQR)) and whiskers showing the range until maximum IQR. The scale on the $y$-axis has been $\log _{10}$ transformed.

two AUCs was not significant $(\mathrm{p}=0.29)$. The NRI was $0.11(11 \% ; \mathrm{p}<0.001)$, which led to a higher specificity. The combination of CRP, PCT and suPAR showed an AUC of 0.91 (95\% CI 0.77-1.00). All AUCs are visualised in figure 3.

A correlation coefficient of 0.1 was found between suPAR and the SAPS II score at admission. SAPS II showed an AUC of 0.57 for diagnosing VAP $(\mathrm{p}=0.42)$. The combination of suPAR and SAPS II resulted in an AUC of 0.71 (95\% CI 0.56-0.87). The difference between these two AUCs was not significant $(\mathrm{p}=0.18)$. The NRI was 0.09 (9\%; $\mathrm{p}=0.002)$, accompanied by an increased specificity.

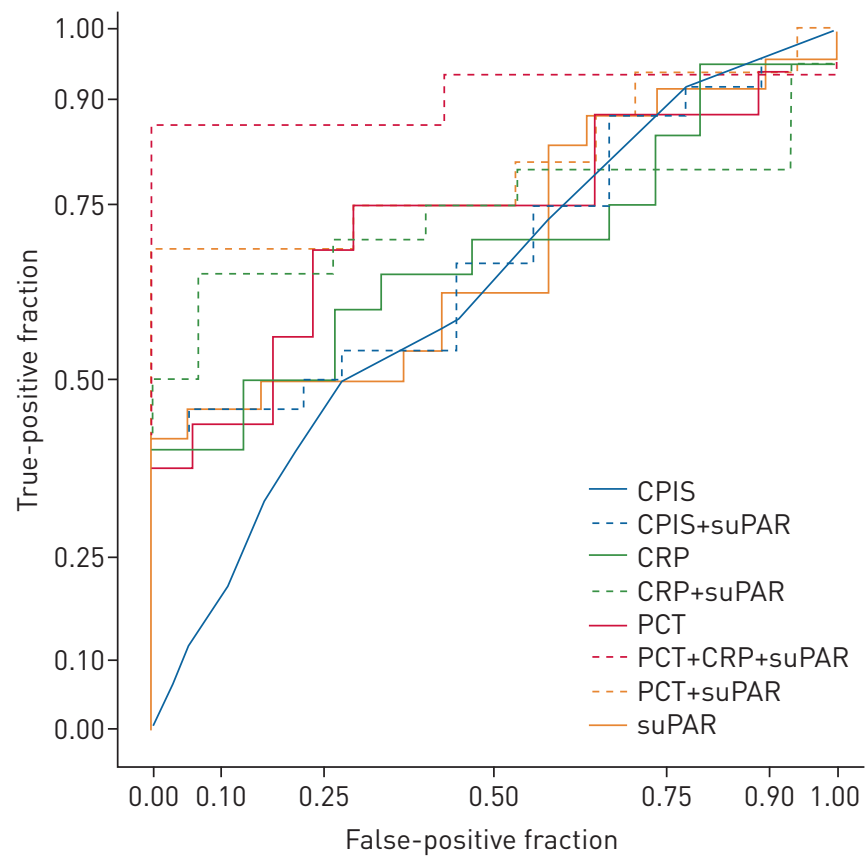

FIGURE 3 Receiver operating characteristic curves for (combinations of) the Clinical Pulmonary Infection Score (CPIS), soluble urokinase plasminogen activator receptor (suPAR), C-reactive protein (CRP) and procalcitonin (PCT): measurements 3 days before ventilator-associated pneumonia diagnosis. 


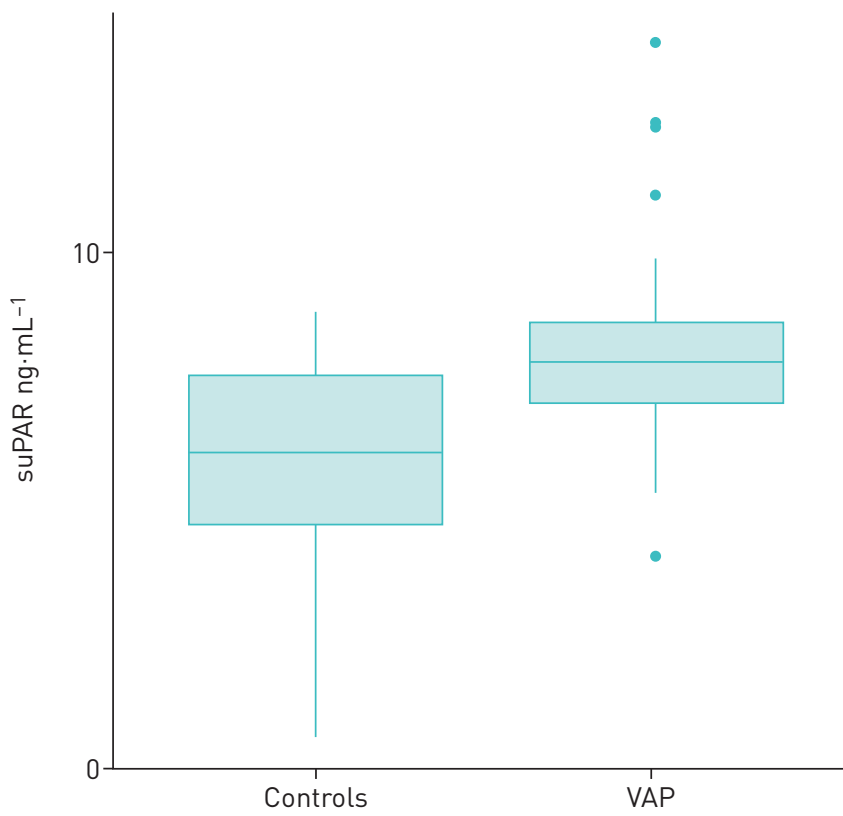

FIGURE 4 Difference in soluble urokinase plasminogen activator receptor (suPAR) levels between diagnosed ventilator-associated pneumonia (VAP) patients and controls, measured on the day of VAP diagnosis. Data are presented as box-and-whisker plots, with boxes showing the median (interquartile range (IQR)) and whiskers showing the range until maximum IQR; outliers are represented by individual data points. The scale on the $y$-axis has been $\log _{10}$ transformed.

\section{Accuracy of sUPAR levels for the diagnosis of VAP}

Also on the day of diagnosis, plasma suPAR levels were elevated in the VAP cases (AUC 0.77, 95\% CI 0.6-0.93; $\mathrm{p}=0.01$ ), with a median (IQR) of $6.6(5.7-7.7) \mathrm{ng} \cdot \mathrm{mL}^{-1}$ for the VAP patients versus $4.7(3.6-6.3) \mathrm{ng} \cdot \mathrm{mL}^{-1}$ for the controls (figure 4). In deceased patients the suPAR levels were significantly higher 3 days before diagnosis (AUC 0.79, 95\% CI 0.57-1.00; $\mathrm{p}<0.001$ ).

On the day of VAP diagnosis, the ROC for the CPIS alone had an AUC of 0.85 (95\% CI 0.74-0.96; $\mathrm{p}=0.001$ ). The AUC of the combination of CPIS plus plasma suPAR was 0.88 (95\% CI 0.77-0.99). The difference between both AUCs was not significant $(\mathrm{p}=0.66)$. When testing this difference using the NRI, the net reclassification improvement was 0.07 (or $7 \%$; $=0.005$ ) when adding the suPAR values to the CPIS, increasing the sensitivity for predicting VAP.

CRP had an AUC of 0.64 (95\% CI 0.47-0.8; $\mathrm{p}=0.09$ ) when measured on the day of diagnosis. CRP combined with suPAR resulted in an AUC of 0.82 (95\% CI $0.69-0.96)$. No significant difference $(\mathrm{p}=0.09)$ was seen between these two AUCs. The NRI was 0.14 (14\%; $\mathrm{p}=0.003)$, leading to an increased sensitivity. A similarly weak positive correlation was found between suPAR and CRP when measured on the day of diagnosis, with a correlation coefficient of 0.23 .

PCT showed an AUC of 0.71 (95\% CI 0.55-0.87; $\mathrm{p}=0.15$ ). The combination of PCT and suPAR had an AUC of 0.85 (95\% CI 0.73-0.97). The difference between the two AUCs was not significant $(\mathrm{p}=0.19)$. An increased sensitivity was seen, with an NRI of $0.12(12 \% ; \mathrm{p}=0.01)$. The combination of CRP, PCT and suPAR resulted in an AUC of 0.88 (95\% CI 0.78-0.99) (figure 5).

The SOFA score and suPAR level, both determined on the day of diagnosis, had a correlation coefficient of 0.15 . The SOFA score could discriminate VAP cases from controls with an AUC of 0.83 (95\% CI 0.71-0.95; $\mathrm{p}=0.003$ ). suPAR combined with the SOFA score led to an AUC of 0.88 (95\% CI 0.78-0.98). The NRI was 0.07 (7\%; $\mathrm{p}=0.02)$, resulting in a higher sensitivity.

\section{Predictive accuracy}

Plasma suPAR levels appeared to be significantly different between alive and deceased patients, both when measured 3 days before diagnosis $(p=0.005)$ and on the day of diagnosis $(p=0.04)$ (figure 6).

Figure 7 shows the association between measured plasma suPAR levels and ICU LOS, showing an OR of $1.43(\mathrm{p}=0.02)$. The association between plasma suPAR and hospital LOS appeared not to be significant (OR 1.0; $\mathrm{p}=0.99)$. 


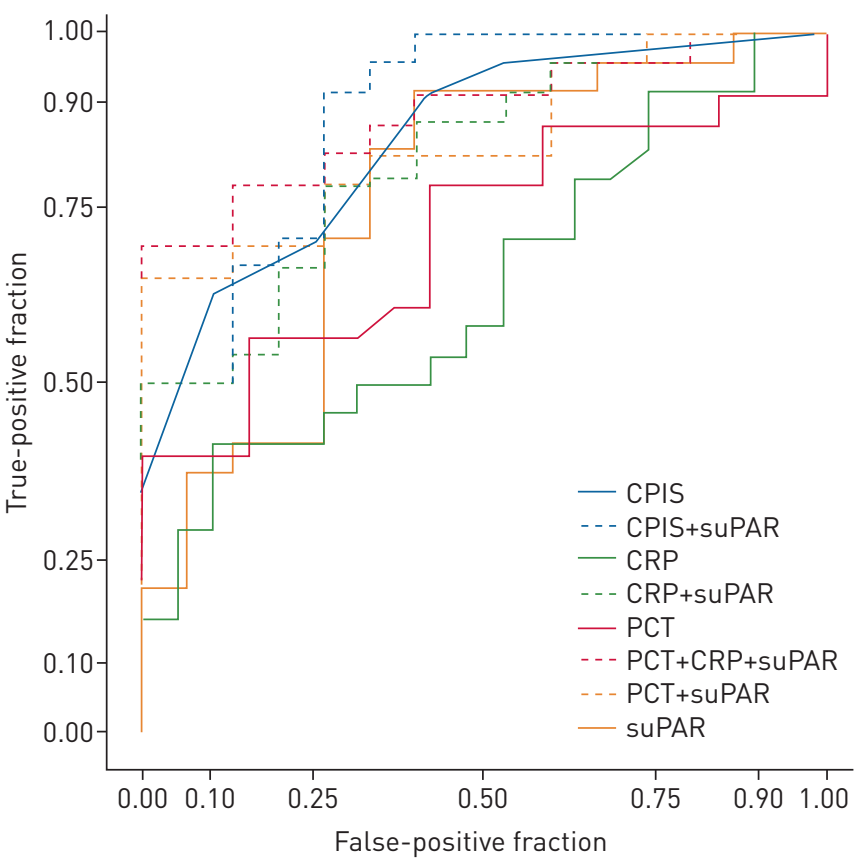

FIGURE 5 Receiver operating characteristic curves for (combinations of) the Clinical Pulmonary Infection Score (CPIS), soluble urokinase plasminogen activator receptor (suPAR), C-reactive protein (CRP) and procalcitonin (PCT): measurements on the day of ventilator-associated pneumonia diagnosis.

\section{Discussion}

Based on the results from this study we conclude that plasma suPAR levels are elevated 3 days before the diagnosis of VAP and have a moderate predictive accuracy. Classification improves when combined with CPIS, CRP and PCT. The diagnostic accuracy was also moderate and does not seem to be sufficient to justify further evaluation. We confirmed moderate prognostic accuracy of suPAR in critically ill ICU patients.

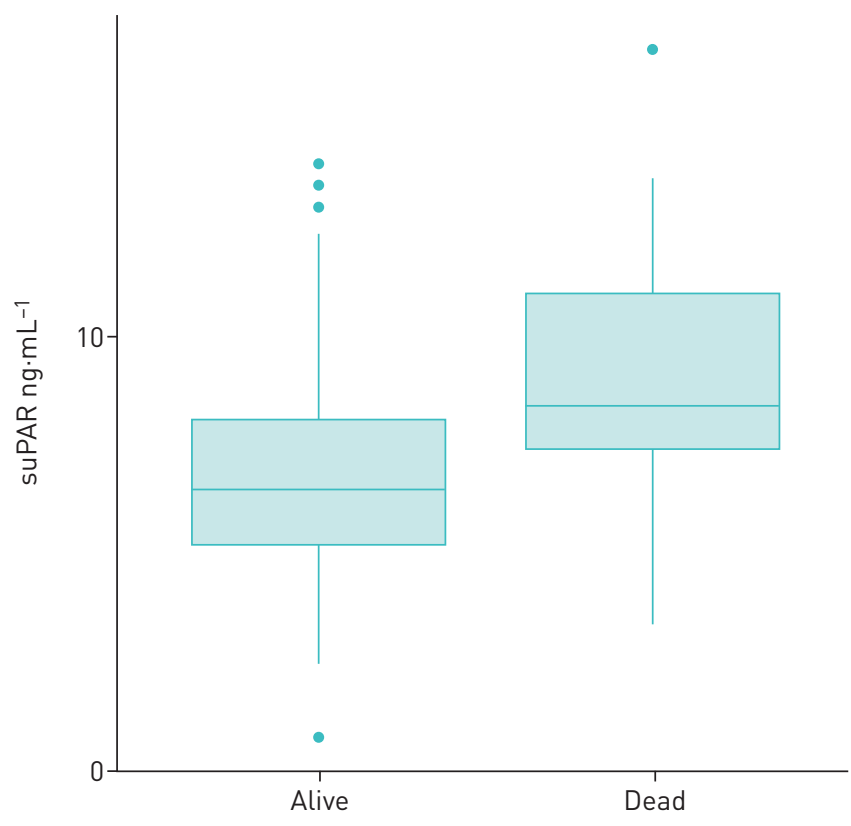

FIGURE 6 Difference in soluble urokinase plasminogen activator receptor (suPAR) levels between alive and deceased patients, measured 3 days before ventilator-associated pneumonia diagnosis. Data are presented as box-and-whisker plots, with boxes showing the median (interquartile range (IQR)) and whiskers showing the range until maximum IQR; outliers are represented by individual data points. The scale on the $y$-axis has been $\log _{10}$ transformed. 


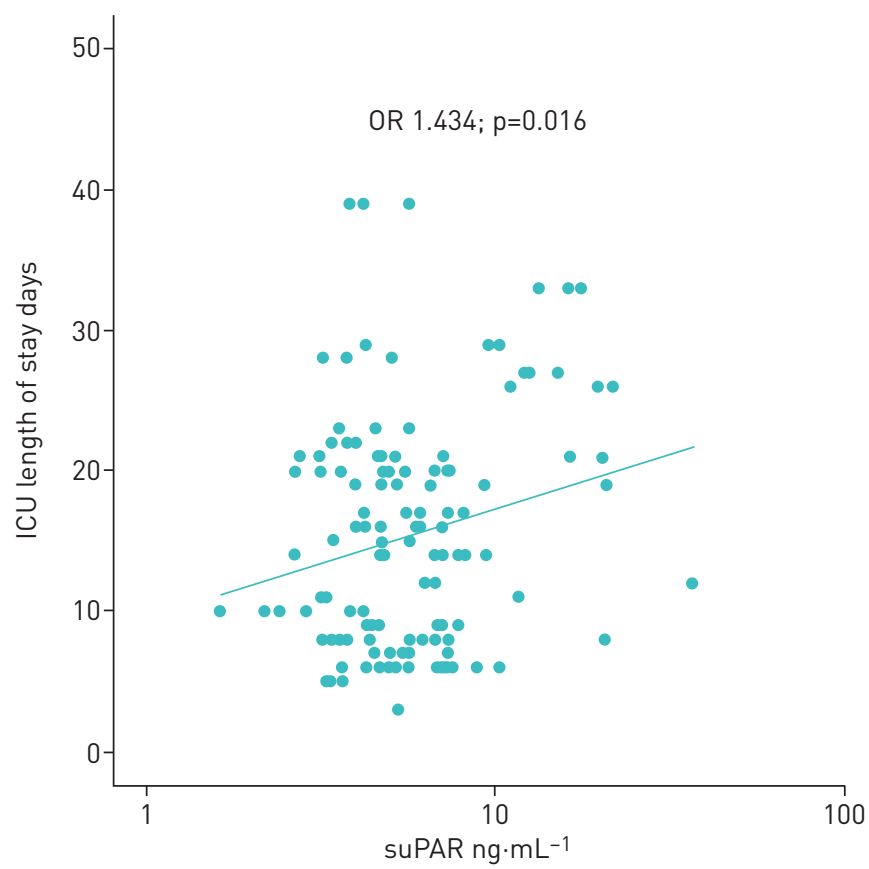

FIGURE 7 Scatterplot showing the association between plasma soluble urokinase plasminogen activator receptor (suPAR) levels and intensive care unit (ICU) length of stay.

Our data suggest that a plasma biomarker may have limited value when used by itself, but its performance can increase substantially when added to a clinical scoring system. In general, biomarkers can be used for diagnostic purposes, response prediction or therapeutic monitoring [26]. The relevance of plasma suPAR may be in its additive predictive value on top of clinical judgement and/or in addition to a scoring system like CPIS, or when used in conjunction with other biomarkers [27]. PCT has been investigated extensively regarding its value to be used as a biomarker to guide antibiotic stewardship [28-32]. As yet, PCT seems to have a poor predictive performance for VAP [12]. Furthermore, the utility of CRP as a marker for VAP has not yet been demonstrated either [33]. Nevertheless, CRP has been used for indication of emerging VAP infection [34] and was used to monitor response to antibiotic treatment in VAP patients [12].

Irrespective of initiation of the appropriate treatment, suPAR remains stable in the systemic circulation of survivors as well as nonsurvivors within the first 10 days of the disease course [19]. The elevated plasma suPAR concentration remains robust for days to weeks, with a half-life of $>7-10$ days [35]. Our results are in accordance with those findings: hardly any change was seen between the suPAR concentration 3 days before and on the day of VAP diagnosis, neither for the VAP patients nor for the control group. As a result, plasma suPAR seems ineligible to evaluate therapy response [36] or guide antibiotic stewardship in VAP. Nevertheless, it could serve as a good early predictor for the disease and thus should be regarded as an extra indicator to distinguish patients at risk of developing VAP.

Serum suPAR has been shown to be associated with disease severity (ICU admission and/or mortality) in patients with community-acquired pneumonia [37,38] and VAP [20]. suPAR levels also seemed to be important as a possible predictor of mortality in systemic inflammatory response syndrome [39]. In our cohort, plasma suPAR levels, when measured 3 days before VAP diagnosis, appeared to have a fair predictive value for mortality in critically ill patients. While there was no association between suPAR and hospital LOS, suPAR levels showed a good correlation with ICU LOS. As the plasma concentration of suPAR reflects immune activation, a recent meta-analysis assessed the usefulness of suPAR for the prognosis of bacterial infections [40]. After pooling the data, a fair performance was found for suPAR in terms of mortality prediction, with an AUC of 0.77 . Regarding the potential of suPAR for diagnosing infection, the same meta-analysis found a pooled sensitivity and specificity of 0.73 and 0.79 , respectively, accompanied by an AUC of 0.82 [40]. An earlier study investigating the diagnostic potential of suPAR for diagnosing VAP reported AUCs of 0.45 for suPAR levels on the first day of mechanical ventilation and 0.25 for suPAR levels within $24 \mathrm{~h}$ after VAP diagnosis [27]. The discrepancy between those results and the AUCs found in our study might be explained in part by the different composition of the control groups. The control group in the study by SunNeTCIOGLu et al. [27] consisted of non-VAP patients that were matched based on age and sex, whereas the control group in our study consisted of non-VAP patients who were matched based on days of mechanical ventilation. 
Within the pathophysiology of pneumonia, Gram-negative or Gram-positive bacteria induce upregulated expression of UPAR (from which suPAR is derived) on monocytes and neutrophils, contributing to the host defence against pulmonary infection, especially demonstrated for respiratory infections caused by $P$. aeruginosa [41]. Reduced neutrophil migration and impaired phagocytosis in the lungs have been shown to occur in uPAR knockout mice; thus, patients with an impaired suPAR system may show a decreased effect of the neutrophil-mediated bactericide reaction [42]. Due to this potential affinity of suPAR with pulmonary processes in particular, it might be specifically interesting (according to our study results) to add suPAR to currently available clinical biomarkers like CRP and PCT or clinical scoring systems like CPIS and SOFA.

Our study investigated the use of suPAR concentrations measured in blood plasma. Alternatively, suPAR can be determined in various other body fluids, e.g. in urine, cerebrospinal fluid or cystic fluid. When measured locally in pleural effusion fluid, suPAR has been shown to be potentially useful for exclusion of infection in ICU patients [43]. suPAR concentrations detected in saliva appeared not to be correlated to suPAR levels in simultaneously obtained plasma samples [44], indicating that care should be taken when directly comparing suPAR values acquired by analysis of fluid samples of different origin.

The strength of this study is in the multicentre and prospective nature of its design, collecting data for 6 days from all noninfected patients that were mechanically ventilated for $>72 \mathrm{~h}$. Limitations include the small sample size of only 43 patients and the absence of a validation cohort. Another limitation is the absence of available suPAR values in patients who were (in part) clinically suspicious for VAP but who had VAP negative or subthreshold microbiology results, since suPAR was only measured in patients who had positive endotracheal aspirate results. However, we could argue that the CPIS captures this clinical suspicion of VAP in a composite of clinical variables and we have been able to show the additive value of suPAR to this clinical score. In addition, the absence of a clinically available gold standard for VAP diagnosis is an important limitation of the study: microbiological examination of BALF is currently the best available reference standard and is, as such, widely accepted by the research community. Future studies into the function of plasma suPAR as a diagnostic biomarker for VAP should use an independent cohort to validate our aforementioned findings.

To conclude, suPAR can be used to diagnose VAP with a fair diagnostic accuracy and has a moderate prognostic accuracy to be used in critically ill ICU patients. Its performance improves when added to other clinically available biomarkers (CRP and PCT) or scoring systems (CPIS and SOFA).

Conflict of interest: P.M. van Oort has nothing to disclose. L.D. Bos has nothing to disclose. P. Póvoa reports grants from Thermo Fisher Scientific during the conduct of the study. P. Ramirez has nothing to disclose. A. Torres reports lectures for Brahms during the conduct of the study. A. Artigas reports grants from and speaking at a symposium for Thermo Fisher Scientific during the conduct of the study. M.J. Schultz has nothing to disclose. I. Martin-Loeches has nothing to disclose.

\section{References}

1 Torres A, Niederman MS, Chastre J, et al. International ERS/ESICM/ESCMID/ALAT guidelines for the management of hospital-acquired pneumonia and ventilator-associated pneumonia. Eur Respir J 2017; 50: 1700582.

2 Warren DK, Shukla SJ, Olsen MA, et al. Outcome and attributable cost of ventilator-associated pneumonia among intensive care unit patients in a suburban medical center. Crit Care Med 2003; 31: 1312-1317.

3 Melsen W, Rovers M, Groenwold R, et al. Attributable mortality of ventilator-associated pneumonia: a meta-analysis of individual patient data from randomised prevention studies. Lancet Infect Dis 2013; 13: 665-671.

4 Martin-Loeches I, Bos LD, Povoa P, et al. Tumor necrosis factor receptor 1 (TNFRI) for ventilator-associated pneumonia diagnosis by cytokine multiplex analysis. Intensive Care Med Exp 2015; 3: 26.

5 Browne E, Hellyer TP, Baudouin S V, et al. A national survey of the diagnosis and management of suspected ventilator-associated pneumonia. BMJ Open Respir Res 2014; 1: e000066.

6 Martin-Loeches I, Povoa P, Rodríguez A, et al. Incidence and prognosis of ventilator-associated tracheobronchitis (TAVeM): a multicentre, prospective, observational study. Lancet Respir Med 2015; 3: 859-868.

7 Chastre J, Fagon J. State of the art ventilator-associated pneumonia. Am J Respir Crit Care Med 2002; 165 867-903.

8 Gibot S, Cravoisy A, Levy B, et al. Soluble triggering receptor expressed on myeloid cells and the diagnosis of pneumonia. N Engl J Med 2004; 350: 451-458.

9 Conway Morris A, Kefala K, Wilkinson TS, et al. Diagnostic importance of pulmonary interleukin-1 $\beta$ and interleukin-8 in ventilator-associated pneumonia. Thorax 2010; 65: 201-207.

10 Hellyer TP, Morris AC, McAuley DF, et al. Diagnostic accuracy of pulmonary host inflammatory mediators in the exclusion of ventilator-acquired pneumonia. Thorax 2014; 70: 41-47.

11 Hellyer TP, Anderson NH, Parker J, et al. Effectiveness of biomarker-based exclusion of ventilator-acquired pneumonia to reduce antibiotic use (VAPrapid-2): study protocol for a randomised controlled trial. Trials 2016; 17: 318.

12 Póvoa P, Martin-Loeches I, Ramirez P, et al. Biomarker kinetics in the prediction of VAP diagnosis: results from the BioVAP study. Ann Intensive Care 2016; 6: 32.

13 Luyt CE, Combes A, Reynaud C, et al. Usefulness of procalcitonin for the diagnosis of ventilator-associated pneumonia. Intensive Care Med 2008; 34: 1434-1440. 
14 Backes Y, Van Der Sluijs KF, Mackie DP, et al. Usefulness of suPAR as a biological marker in patients with systemic inflammation or infection: a systematic review. Intensive Care Med 2012; 38: 1418-1428.

15 Jalkanen V, Yang R, Linko R, et al. SuPAR and PAI-1 in critically ill, mechanically ventilated patients. Intensive Care Med 2013; 39: 489-496.

16 Geboers DGPJ, de Beer FM, de Boer AMT, et al. Plasma suPAR as a prognostic biological marker for ICU mortality in ARDS patients. Intensive Care Med 2015; 41: 1281-1290.

17 Backes Y, van der Sluijs KF, de Boer AM T, et al. Soluble urokinase-type plasminogen activator receptor levels in patients with burn injuries and inhalation trauma requiring mechanical ventilation: an observational cohort study. Crit Care 2011; 15: R270.

18 Rasmussen LJH, Ladelund S, Haupt TH, et al. Soluble urokinase plasminogen activator receptor (suPAR) in acute care: a strong marker of disease presence and severity, readmission and mortality. A retrospective cohort study. Emerg Med J 2016; 33: 769-775.

19 Koch A, Voigt S, Kruschinski C, et al. Circulating soluble urokinase plasminogen activator receptor is stably elevated during the first week of treatment in the intensive care unit and predicts mortality in critically ill patients. Crit Care 2011; 15: R63.

20 Savva A, Raftogiannis M, Baziaka F, et al. Soluble urokinase plasminogen activator receptor (suPAR) for assessment of disease severity in ventilator-associated pneumonia and sepsis. J Infect 2011; 63: 344-350.

21 Pugin J, Auckenthaler R, Mili N, et al. Diagnosis of ventilator-associated pneumonia by bacteriologic analysis of bronchoscopic and nonbronchoscopic "blind" bronchoalveolar lavage fluid. Am Rev Respir Dis 1991; 143 $1121-1129$.

22 DeLong ER, DeLong DM, Clarke-Pearson DL. Comparing the areas under two or more correlated receiver operating characteristic curves: a nonparametric approach. Biometrics 1988; 44: 837-845.

23 R Development Core Team. R: A Language and Environment for Statistical Computing. Vienna, R Foundation for Statistical Computing, 2010

24 Le Gall J, Lemeshow S, Saulnier F. A new Simplified Acute Physiology Score (SAPS II) based on a European/ North American multicenter study. JAMA 1993; 270: 2957-2963.

25 Vincent J-L, Moreno R, Takala J, et al. The SOFA (Sepsis-related Organ Failure Assessment) score to describe organ dysfunction/failure. Intensive Care Med 1996; 22: 707-710.

26 Selleck MJ, Senthil M, Wall NR. Making meaningful clinical use of biomarkers. Biomark Insights 2017; 12 1177271917715236 .

27 Sunnetcioglu A, Sunnetcioglu M, Adiyaman F, et al. Could soluble urokinase plasminogen receptor (suPAR) be used as a diagnostic biomarker for ventilator-associated pneumonia? Clin Respir J 2017; 11: 925-930.

28 de Jong E, van Oers JA, Beishuizen A, et al. Efficacy and safety of procalcitonin guidance in reducing the duration of antibiotic treatment in critically ill patients: a randomised, controlled, open-label trial. Lancet Infect Dis 2016 16: 819-827.

29 Bloos F, Trips E, Nierhaus A, et al. Effect of sodium selenite administration and procalcitonin-guided therapy on mortality in patients with severe sepsis or septic shock. JAMA Intern Med 2016; 176: 1266-1276.

30 Chu DC, Mehta AB, Walkey AJ. Practice patterns and outcomes associated with procalcitonin use in critically ill patients with sepsis. Clin Infect Dis 2017; 64: 1509-1515.

31 Huang DT, Yealy DM, Filbin MR, et al. Procalcitonin-guided use of antibiotics for lower respiratory tract infection. N Engl J Med 2018; 379: 236-249.

32 Daubin $\mathrm{C}$, Valette $\mathrm{X}$, Thiollière $\mathrm{F}$, et al. Procalcitonin algorithm to guide initial antibiotic therapy in acute exacerbations of COPD admitted to the ICU: a randomized multicenter study. Intensive Care Med 2018; 44 428-437.

33 Palazzo SJ, Simpson T, Schnapp L. Biomarkers for ventilator-associated pneumonia: review of the literature. Hear Lung J Acute Crit Care 2011; 40: 293-298.

34 Filipiak W, Beer R, Sponring A, et al. Breath analysis for in vivo detection of pathogens related to ventilator-associated pneumonia in intensive care patients: a prospective pilot study. J Breath Res 2015; 9: 016004.

35 Huttunen R, Syrjänen J, Vuento R, et al. Plasma level of soluble urokinase-type plasminogen activator receptor as a predictor of disease severity and case fatality in patients with bacteraemia: a prospective cohort study. J Intern Med 2011; 270: 32-40.

36 Donadello K, Scolletta S, Taccone FS, et al. Soluble urokinase-type plasminogen activator receptor as a prognostic biomarker in critically ill patients. J Crit Care 2014; 29: 144-149.

37 Loonen AJM, Kesarsing C, Kusters R, et al. High pneumococcal DNA load, procalcitonin and suPAR levels correlate to severe disease development in patients with pneumococcal pneumonia. Eur J Clin Microbiol Infect Dis 2017; 36: 1541-1547.

38 Luo Q, Ning P, Zheng Y, et al. Serum suPAR and syndecan-4 levels predict severity of community-acquired pneumonia: a prospective, multi-centre study. Crit Care 2018; 22: 1-12.

39 Yilmaz G, Köksal I, Karahan SC, et al. The diagnostic and prognostic significance of soluble urokinase plasminogen activator receptor in systemic inflammatory response syndrome. Clin Biochem 2011; 44: 1227-1230.

$40 \mathrm{Ni}$ W, Han Y, Zhao J, et al. Serum soluble urokinase-type plasminogen activator receptor as a biological marker of bacterial infection in adults: a systematic review and meta-analysis. Sci Rep 2016; 6: 39481 .

41 Rijneveld AW, Levi M, Florquin S, et al. urokinase receptor is necessary for adequate host defense against pneumococcal pneumonia. I Immunol 2002; 168: 3507-3511.

42 Wrotek A, Jackowska T, Pawlik K. Soluble urokinase plasminogen activator receptor: an indicator of pneumonia severity in children. Adv Exp Med Biol 2015; 835: 1-7.

43 Bakker OGM, Hemmes SNT, Backes Y, et al. SuPAR in pleural fluid may function as a biological marker for infection in critically ill patients with pleural effusions. J Infect 2014; 68: 607-609.

44 Gustafsson A, Ajeti V, Ljunggren L. Detection of suPAR in the saliva of healthy young adults: comparison with plasma levels. Biomark Insights 2011; 6: 119-125. 